\title{
Gromov Hyperbolicity in Directed Graphs
}

\author{
Ana Portilla ${ }^{1}$, José M. Rodríguez ${ }^{2, * \mathbb{D}}$, José M. Sigarreta ${ }^{3,4}$ and $^{\text {Eva Tourís }}{ }^{5}$ (D) \\ 1 Math and Computer Science Department, St. Louis University (Madrid Campus), Avenida del Valle 34, \\ 28003 Madrid, Spain; ana.portilla@slu.edu \\ 2 Departamento de Matemáticas, Universidad Carlos III de Madrid, Avenida de la Universidad 30, \\ 28911 Leganés, Madrid, Spain \\ 3 Instituto de Física, Benemérita Universidad Autónoma de Puebla, Apartado Postal J-48, \\ Puebla 72570, Mexico; josemariasigarretaalmira@hotmail.com \\ 4 Facultad de Matemáticas, Universidad Autónoma de Guerrero, Carlos E. Adame No.54 Col. Garita, \\ Acapulco Gro. 39650, Mexico \\ 5 Departamento de Matemáticas, Facultad de Ciencias, Universidad Autónoma de Madrid, \\ Campus de Cantoblanco, 28049 Madrid, Spain; eva.touris@uam.es \\ * Correspondence: jomaro@math.uc3m.es; Tel.: +34-916-249-098
}

Received: 22 November 2019; Accepted: 1 January 2020; Published: 6 January 2020

\begin{abstract}
In this paper, we generalize the classical definition of Gromov hyperbolicity to the context of directed graphs and we extend one of the main results of the theory: the equivalence of the Gromov hyperbolicity and the geodesic stability. This theorem has potential applications to the development of solutions for secure data transfer on the internet.
\end{abstract}

Keywords: security; Gromov hyperbolicity; hyperbolic graph; directed graph

\section{Introduction}

A geodesic metric space is said to be (Gromov) hyperbolic if every geodesic triangle is uniformly "thin" (see Definition 2). The theory of hyperbolic spaces was introduced by M. Gromov in [1,2], and after that studied by many scientists, see, e.g., in [3-6]. It is especially remarkable that this "new" theory grasps the essence of negatively curved spaces, and the connections between graph theory and potential theory on Riemannian manifolds (see, e.g., in [7-11]).

In [12], a certain graph is constructed for each geodesic metric space and it is proved that one is hyperbolic if and only if the other is; furthermore, in [13-15] there are analogous results for Riemannian surfaces (and simple graphs). Therefore, the study of Gromov hyperbolic graphs can shed light on the hyperbolicity of more general geodesic metric spaces.

The research on mathematical properties of hyperbolic spaces and their applications is a subject of interest in graph theory; see, e.g., in [16-37] and the references therein.

Originally, the theory of hyperbolic spaces was developed to be applied to finitely generated groups and, in that field, it has proved relevant competence in practical applications. For example, they were historically applied to some computer science questions, as hyperbolic groups have a soluble word problem. In [38], it is proved that they are biautomatic and automatic; actually, they are strongly geodesically automatic, meaning that there is an automatic structure on the group, where the set of all geodesic words is the language accepted by the word acceptor. Many other applications are supported by the concept of Gromov hyperbolicity. For instance, research evidence suggests that the Internet is hyperbolic (see [39]); the outstanding growth/preferential attachment process as a mean to construct a scale-free graph produces a (scaled) hyperbolic graph (see [28,40]); the greedy geographical routing tries to embed the network graph in the (hyperbolic) Poincare disk (see [41]). The spread of viruses through the Internet can be modeled by hyperbolic spaces (see $[27,42])$. 
Today, and thanks to the geodesic stability of hyperbolic spaces (see Theorems 2 and 3), Gromov hyperbolicity is successfully applied to the development of solutions for secure data transfer on the internet. As Edmond Jonckheere states in [42], any electronic message is usually split into many different packets, which are sent separately to its destination. Most of them follow the same path, but, unfortunately, it facilitates the interception and subsequent reconstruction of the message by malicious listeners. A better strategy to bypass this type of electronic spying is to send packets along distinct routes [43]. However, this design raises a further difficulty: the reconstruction by the protocol of all those packets, which reach destination in a disorganized way (due to the intrinsic delays of the different routes chosen), is not trivial.

For all these reasons, a remarkable feature of hyperbolic networks is that in a (well-located by geodesic stability) neighborhood of any geodesic (optimal path) there are many other quasi-optimal paths, i.e., routes whose costs are close to the optimal one. Thus, hyperbolic networks facilitate the assembly of packets sent through different routes with similar delay times [44].

Nevertheless, when it comes to the application of Gromov spaces to this field, some problems arise due to the fact that modelization still not accurate of this process is not yet accurate. The communication between a client computer and its internet connection server is not "symmetric" in the following sense: data transferring from server to client is much faster than from client to server. Mathematically, it means that the function modeling the distance between client and server is not symmetric either, i.e., it is not a distance function.

We can first address the problem with a mathematical model that assumes that data transferring between server and client is symmetric. Although we know that this is not very accurate, it seems a very reasonable approach, as this makes it possible to work with a usual distance function (see the discussion in [42]).

However, it is desirable to pursue a more realistic model. For instance, Edmond Jonckheere cleverly decided to apply noncommutative geometry methods to overcome the difficulty introduced by the asymmetry (see [42]).

Our main aim in this paper is to show a new mathematical theory to provide an alternative approach to the problem, involving a less sophisticated mathematical foundation. In particular, we think that a more intuitive model can be found in "directed graphs": each pair of adjacent vertices $A$ and $B$ is joined by two directed edges $A B$ and $B A$ of (possibly) different lengths. This point of view introduces asymmetry as something natural.

In real life asymmetric distances are natural; for instance, given a set $X$ of mountain peaks connected by twisted roads, the usual driving times between these peaks form a quasi-metric because it takes longer to travel up hill than down hill; also, the taxi geometry with some one-way streets, where a path from location $P$ to location $Q$ contains a different set of streets than a path from $Q$ to $P$.

Thus, in this paper we extend Gromov hyperbolic spaces to the new context of directed graphs. Specifically, we prove one of the most important results of the theory: a counterpart to the geodesic stability theorem, which happens to be the most utilized theorem both in theory and in applications of hyperbolic spaces in the usual context of geodesic metric spaces (see Theorem 5).

Notation. For each geodesic metric space $X$, we shall denote by $d_{X}$ and $L_{X}$, the distance and the length, respectively.

\section{Background on Hyperbolic Spaces}

Although there are several definitions of hyperbolicity, in this paper we will use the one that involves thin triangles, as it is intuitively simple and has a clear geometric interpretation. For more background and results on hyperbolicity, see [2].

Definition 1. If $X$ is a metric space and $I$ is a real interval, then a curve $\gamma: I \rightarrow X$ is a geodesic if it is an isometry, i.e., $L_{X}\left(\left.\gamma\right|_{[t, s]}\right)=d_{X}(\gamma(t), \gamma(s))=|t-s|$ for each $t, s$ in the interval $I$. $X$ is said to be a geodesic 
metric space if for each $x_{1}, x_{2} \in X$ there exists at least one geodesic joining $x_{1}$ and $x_{2}$; we denote by $\left[x_{1} x_{2}\right]$ any of such geodesics (since uniqueness of geodesics is not required, this is an ambiguous but convenient notation).

Definition 2. If $X$ is a geodesic metric space and $Y=\left\{Y_{1}, Y_{2}, Y_{3}\right\}$, with $Y_{j} \subseteq X$, we say that $Y$ is $\delta$-thin if for each $j \in\{1,2,3\}$ and $y \in Y_{j}$ one has $d\left(Y, \cup_{i \neq j} Y_{i}\right) \leqslant \delta$. If $x, y, z \in X$, then a geodesic triangle $T=\{x, y, z\}$ is the union of the three geodesics $[x y],[y z]$ and $[z x] . X$ is said to be $\delta$-hyperbolic (or that it satisfies the Rips condition) if each geodesic triangle in $X$ is $\delta$-thin. Denote by $\delta(X)$ the sharp hyperbolicity constant of the space $X$, i.e., $\delta(X)=\inf \{\delta \geqslant 0: X$ is $\delta$-hyperbolic $\}$. The space $X$ is said to be Gromov hyperbolic (or simply hyperbolic) if it is $\delta$-hyperbolic for some $\delta \geqslant 0$. If $X$ is not hyperbolic, then define $\delta(X)=\infty$.

\section{Example 1.}

I Each bounded space $S$ is (diam S)/2-hyperbolic [2] (p. 29).

II Any complete simply connected manifold with sectional curvature above bounded by $-k^{2}<0$ is hyperbolic (see, e.g., in [45] (p. 130) and [2] (p. 52)).

III Every tree with edges of arbitrary length is 0-hyperbolic (see, e.g., in [2] (p. 29)).

In fact, the hyperbolicity constant $\delta(X)$ of a geodesic metric space can be interpreted as a way to decide how far the space is from being a tree. Recall that the metric trees are the spaces with hyperbolicity constant equal to zero. In practice, the borderline between tractable and intractable problems can be the tree-like degree of the corresponding graph (see [46]).

Next, the class of maps which play a central role in the theory is presented.

Definition 3. Given constants $a \geqslant 1, b \geqslant 0$, a function between two metric spaces $f: X \longrightarrow Y$ is said to be an $(a, b)$-quasi-isometry if

$$
\frac{1}{a} d_{X}\left(x_{1}, x_{2}\right)-b \leqslant d_{Y}\left(f\left(x_{1}\right), f\left(x_{2}\right)\right) \leqslant a d_{X}\left(x_{1}, x_{2}\right)+b, \quad \text { for every } x_{1}, x_{2} \in X .
$$

$A n(a, b)$-quasigeodesic in $X$ is an $(a, b)$-quasi-isometry from a real interval to $X$.

Next result shows that quasi-isometries preserve hyperbolicity. That is why they play a central role in Gromov hyperbolic spaces.

Theorem 1. ([2] (p. 88)) Let us consider an $(a, b)$-quasi-isometry between two geodesic metric spaces $f$ : $X \longrightarrow Y$. If $Y$ is hyperbolic, then $X$ is hyperbolic. Furthermore, if $f$ is onto, then $X$ is hyperbolic if and only if $Y$ is hyperbolic.

Definition 4. Let us consider $H>0$, a metric space $X$, and subsets $Y, Z \subseteq X$. The set $V_{H}(Y):=\{x \in X$ : $d(x, Y) \leqslant H\}$ is called the $H$-neighborhood of $Y$ in $X$. The Hausdorff distance between $Y$ and $Z$ is defined by $\mathcal{H}(Y, Z):=\inf \left\{H>0: Y \subseteq V_{H}(Z), Z \subseteq V_{H}(Y)\right\}$.

The following is a beautiful and useful result:

Theorem 2. ([2] ( $p .87))$ For each $\delta \geqslant 0, a \geqslant 1$ and $b \geqslant 0$, there exists a constant $H=H(\delta, a, b)$, which only depends on these three parameters, with the following property:

Let us consider any $\delta$-hyperbolic geodesic metric space $X$, any $(a, b)$-quasigeodesic $g$ joining $x$ and $y$. If $\gamma$ is any geodesic joining $x$ and $y$, then $\mathcal{H}(g, \gamma) \leqslant H$.

Mario Bonk proved that the above property, known as "geodesic stability", is equivalent to hyperbolicity, as shown below.

Theorem 3. ([47] (p. 286)) Let us consider a geodesic metric space X with the following property: 
For each $a \geqslant 1$ there exists a constant $H$ such that for any $x, y \in X$, any $(a, 0)$-quasigeodesic $g$ in $X$ joining $x$ and $y$, and any geodesic $\gamma$ joining $x$ and $y$, the inequality $\mathcal{H}(g, \gamma) \leqslant H$ holds.

Then, $X$ is hyperbolic.

Note that Theorems 2 and 3 give that, in fact, the hypothesis in Theorem 3 is equivalent to the following: For each $a \geqslant 1$ and $b \geqslant 0$ there exists a constant $H$ such that for any $x, y \in X$, any $(a, b)$-quasigeodesic $g$ in $X$ joining $x$ and $y$, and any geodesic $\gamma$ joining $x$ and $y$, the inequality $\mathcal{H}(g, \gamma) \leqslant H$ holds.

\section{Directed Graphs}

If $G=(V(G), E(G))$ is a directed (finite or infinite) graph, with $V(G)$ the set of vertices and $E(G)$ the edges, we use the notation $u v$ for an edge starting at a vertex $u$ and ending at the vertex $v$. As we allow loops and multiple edges this notation is ambiguous, but it is convenient. We suppose that $G$ is locally finite, i.e., each vertex belongs to a finite amount of edges. Also, assume that each edge $u v$ has length $L_{G}(u v)$. We shall identify (by an isometry) each directed edge $u v \in E(G)$ with the directed real interval $[0, l]$ (where $l:=L_{G}(u v)$ ); therefore, any point in the interior of the edge $u v$ is a point of $G$, and then $G$ is a metric directed graph.

Definition 5. We say that a directed graph is admissible if it is connected (for every $x, y \in G$ there exists a directed path from $x$ to $y$ ), locally finite (i.e., every vertex has finite degree) and we have $u v \in E(G)$ if and only if $v u \in E(G)$ (we allow $L_{G}(u v) \neq L_{G}(v u)$ ). (See Figure $1 a$.)

Given an admissible graph $G$ and two adjacent vertices $u, v \in V(G)$, let us define the parameters

$$
\begin{aligned}
C(u, v) & :=\frac{\max \left\{L_{G}(e): e \text { is an edge joining either } u \text { and } v \text { or } v \text { and } u\right\}}{\min \left\{L_{G}(e): e \text { is an edge joining either } u \text { and } v \text { or } v \text { and } u\right\}}, \\
C(G) & :=\sup \{C(u, v)\}, \\
D(G) & :=\sup \left\{L_{G}(e): e \in E(G)\right\} .
\end{aligned}
$$

Note that $1 \leqslant C(u, v) \leqslant C(G) \leqslant \infty$ and $0<D(G) \leqslant \infty$.

Definition 6. Given an admissible graph $G$, we define its canonical graph $G_{0}$ as the simple (without loops and multiple edges) undirected graph with $V\left(G_{0}\right)=V(G)$ obtained by replacing the directed edges joining two adjacent vertices $u$ and $v$ in $G$ by a single undirected edge, $u v \in E\left(G_{0}\right)$, with

$$
L_{G_{0}}(u v):=\max \left\{L_{G}(e): e \text { is an edge joining either } u \text { and } v \text { or } v \text { and } u\right\} .
$$

It can be checked that $G_{0}$ is a geodesic metric space; thus, we also see $G_{0}$ as a metric graph (see Figure 1b).

Remark 1. Note that if $C(G)<\infty$, then for any edge $e \in E(G)$ joining $u$ and $v$ (or $v$ and $u$ ) it holds

$$
L_{G}(e) \leqslant L_{G_{0}}(u v) \leqslant C(G) L_{G}(e) .
$$

Although there is no usual distance in an admissible directed graph $G$, we introduce now the following quasi-distance or quasi-metric $D_{G}$ :

$$
D_{G}(a, b):=\inf \left\{L_{G}(\eta): \eta \text { is a directed curve starting at } a \text { and ending at } b\right\} .
$$

Recall that a quasi-distance satisfies the properties of a distance except for the symmetry, e.g., it is possible to have $D_{G}(a, b) \neq D_{G}(b, a)$. 
However, we have the following relationship between $D_{G}(u, v)$ and $D_{G}(v, u)$ : if $C(G)<\infty$, then

$$
\frac{1}{C(G)} D_{G}(u, v) \leqslant D_{G}(v, u) \leqslant C(G) D_{G}(u, v), \quad \text { for every } u, v \in V(G) .
$$

Quasi-metrics are used in many contexts, such as Computer Science and Approximation Theory. From a historical viewpoint, a kind of quasi-metric spaces were already considered for the first time by Hausdorff in his book on Set Theory [48]. This concept is extended later to introduce quasi-uniform topological spaces. Other authors also studied asymmetric distances. In [49], Künzi claims that there was much progress in quasi-uniform spaces between the years 1966 and 1982. This is just the period when different authors gave the first steps in asymmetric approximation. Indeed, to the intrinsic interest of this topic we might add applications in Approximation Theory and Computer Science. That is why there exists a vast amount of results both on abstract asymmetric normed spaces and on more general quasi-metric spaces.

One of the main applications in Computer Science is the project to develop robust models for measuring the complexity distance between programs and algorithms, which has been considered in the last 40 years. A model in the context of quasi-metric spaces was introduced by M. Schellekens in [50] and further developed by different authors (see, e.g., [51] and the references therein).

Definition 7. Given constants $a \geqslant 1, b \geqslant 0$, we say that a function between an interval I of $\mathbb{R}$ and $a$ directed graph $f: I \longrightarrow G$ is an $(a, b)$-quasigeodesic if

$$
\frac{1}{a}(t-s)-b \leqslant D_{G}(f(s), f(t)) \leqslant a(t-s)+b, \quad \text { for every } s, t \in I, \text { with } s \leqslant t .
$$

A geodesic in a directed graph $\left(G, D_{G}\right)$ is a $(1,0)$-quasigeodesic in $\left(G, D_{G}\right)$. We denote by $[x y]$ a geodesic starting at $x$ and ending at $y$. (See Figure 1).

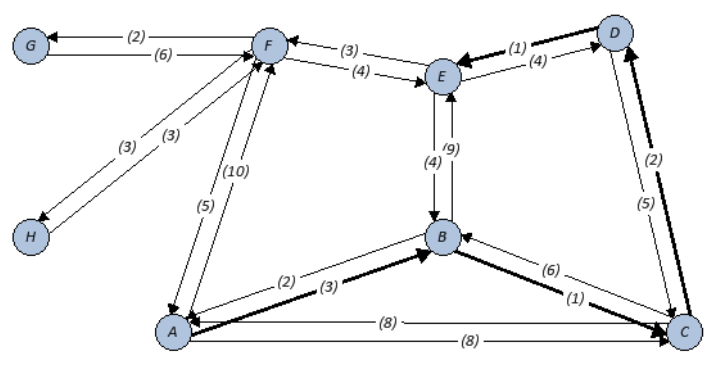

(a)

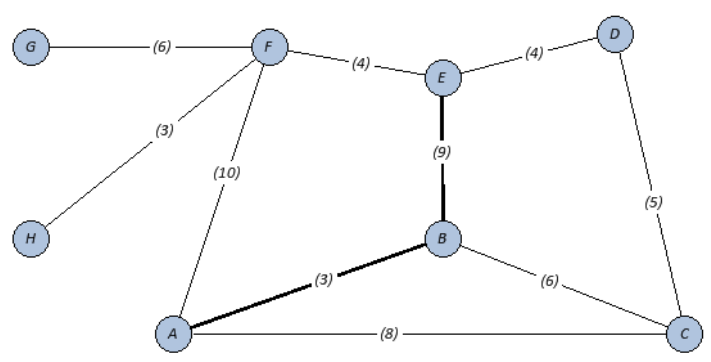

(b)

Figure 1. (a) An admissible graph $G$. (In bold face, the directed geodesic joining $A$ and $E$ ); (b) Its canonical graph $G_{0}$. (In bold face, the geodesic joining $A$ and $E$ ). 
Next, let us define the Hausdorff distance in any directed graph $G$.

Definition 8. Let us consider a directed graph $G$, and subsets $A, B \subseteq G$. We define the "Hausdorff distance", $\mathcal{H}_{G}(A, B)$, between $A$ and $B$ in $G$ as the infimum of the positive numbers $\alpha$ satisfying the two following properties:

I For every $a \in A$ there exists $b \in B$ such that $D_{G}(a, b) \leqslant \alpha$ and $D_{G}(b, a) \leqslant \alpha$.

II For every $b \in B$ there exists $a \in A$ such that $D_{G}(b, a) \leqslant \alpha$ and $D_{G}(a, b) \leqslant \alpha$.

Note that $\mathcal{H}_{G}$ is a symmetric function although $D_{G}$ is, in general, non-symmetric.

Definition 9. Given an admissible directed graph $G$ such that $C(G), D(G)$ are finite numbers, we say that $G$ is $\delta$-hyperbolic if and only if its canonical graph $G_{0}$ is $\delta$-hyperbolic. We say that $G$ is hyperbolic or Gromov hyperbolic if it is $\delta$-hyperbolic for some $\delta \geqslant 0$.

To prove the following theorem we need two last definitions.

Definition 10. If $G$ is an admissible directed graph and $x_{1}, x_{2}, x_{3} \in G$, a geodesic triangle $T=\left\{x_{1}, x_{2}, x_{3}\right\}$ in $G$ is the union of three directed geodesics $J_{1}=\left[x_{1} x_{2}\right]$ (or $\left.\left[x_{2} x_{1}\right]\right), J_{2}=\left[x_{2} x_{3}\right]\left(\right.$ or $\left[x_{3} x_{2}\right]$ ) and $J_{3}=$ $\left[x_{3} x_{1}\right]$ (or $\left[x_{1} x_{3}\right]$ ). We say that $T$ is $\delta$-thin if for every $x \in J_{i}$ there exists a point $y \in \cup_{j \neq i} J_{j}$ such that $D_{G}(x, y), D_{G}(y, x) \leqslant \delta$.

Definition 11. If $u v$ is an edge in the admissible directed graph $G$ (or in the graph $G_{0}$ ) and $x \in u v$, we denote by $\overline{u x}$ (respectively, $\overline{x v}$ ) the segment contained in $u v$ starting at $u$ and ending at $x$ (respectively, starting at $x$ and ending at $v)$. Let us define the canonical projection $\Pi: G \rightarrow G_{0}$ as follows: $\Pi(v)=v$ for every $v \in V(G)$; if $x \in u v \in E(G)$, then $\Pi(x)$ is the point in $u v \in E\left(G_{0}\right)$ such that

$$
L_{G_{0}}(\overline{u \Pi(x)})=L_{G}(\overline{u x}) \frac{L_{G_{0}}(u v)}{L_{G}(u v)} .
$$

The following result shows that we have a reasonable definition of Gromov hyperbolic directed graphs.

Theorem 4. Any geodesic triangle $T$ in a $\delta$-hyperbolic admissible directed graph $G$ with $C(G), D(G)$ finite numbers is $\delta^{\prime}$-thin, with $\delta^{\prime}$ a constant which only depends on $\delta, C(G)$ and $D(G)$.

Proof. We perform the proof in three steps. We first show that given any geodesic $\gamma$ in $G$, then $\Pi(\gamma)$ is a $(C(G), 4 D(G))$-quasigeodesic in $G_{0}$. Then, we prove that every $(a, b)$-quasigeodesic triangle in $G_{0}$ is $(\delta+2 H(\delta, a, b))$-thin, where $H(\delta, a, b)$ is the constant in Theorem 2 . These facts will allow us to conclude that every geodesic triangle in $G$ is $\delta^{\prime}$-thin, with $\delta^{\prime}=\delta+2 H(\delta, C(G), 4 D(G))+4 D(G)$.

Step 1. From (1) we can deduce that

$$
D_{G}(x, y) \leqslant d_{G_{0}}(\Pi(x), \Pi(y)) \leqslant C(G) D_{G}(x, y) \quad \text { for every } x, y \in V(G) .
$$

If $u \in V(G)$ and $x$ belongs to some edge starting or ending at $u$, then $D_{G}(x, u), D_{G}(u, x) \leqslant 2 D(G)$; this fact and (2) allows to deduce

$$
D_{G}(x, y)-4 D(G) \leqslant d_{G_{0}}(\Pi(x), \Pi(y)) \leqslant C(G) D_{G}(x, y) \quad \text { for every } x, y \in G .
$$

Therefore, if $\gamma$ is a geodesic in $G$ starting at $x$ and ending at $y$, according to (3), we have that $\Pi(\gamma)$ is a $(C(G), 4 D(G))$-quasigeodesic in $G_{0}$ joining $\Pi(x)$ and $\Pi(y)$. 
Step 2. Let us consider an $(a, b)$-quasigeodesic triangle $T^{\prime}$ in $G_{0}$, whose sides we will denote by $g_{1}, g_{2}$ and $g_{3}$. Let $\gamma_{j}$ be the geodesic in $G_{0}$ joining the same endpoints of $g_{j}$ for every $j=1,2,3$, and let us denote by $T_{0}$ the geodesic triangle in $G_{0}$ with sides $\gamma_{1}, \gamma_{2}, \gamma_{3}$.

Let us consider any point $p^{\prime} \in T^{\prime}$; without lost of generality we can assume that $p^{\prime} \in g_{1}$. By Theorem 2 we know that there exist a constant $H=H(\delta, a, b) \geqslant 0$ and a point $p_{0} \in \gamma_{1}$ with $d_{G_{0}}\left(p^{\prime}, p_{0}\right) \leqslant H$. As $T_{0}$ is $\delta$-thin, there exists $q_{0} \in \gamma_{2} \cup \gamma_{3}$ with $d_{G_{0}}\left(p_{0}, q_{0}\right) \leqslant \delta$. According to Theorem 2, there exists a point $q^{\prime} \in g_{2} \cup g_{3}$ with $d_{G_{0}}\left(q^{\prime}, q_{0}\right) \leqslant H$.

Therefore,

$$
d_{G_{0}}\left(p^{\prime}, g_{2} \cup g_{3}\right) \leqslant d_{G_{0}}\left(p^{\prime}, q^{\prime}\right) \leqslant d_{G_{0}}\left(p^{\prime}, p_{0}\right)+d_{G_{0}}\left(p_{0}, q_{0}\right)+d_{G_{0}}\left(q_{0}, q^{\prime}\right) \leqslant H+\delta+H,
$$

and $T^{\prime}$ is $(\delta+2 H(\delta, a, b))$-thin.

Step 3. Consequently, if $T$ is a geodesic triangle in $G$, by Step 1 we have that $T^{\prime}:=\Pi(T)$ is a $(C(G), 4 D(G))$-quasigeodesic triangle in $G_{0}$, and by Step 2 we know that $T^{\prime}$ is $(\delta+2 H)$-thin with $H=H(\delta, C(G), 4 D(G))$. Let us prove now that $T$ is $\delta^{\prime}$-thin in $G$.

Given any point $p \in T$, there exists a point $q$ in a different side of $T$ such that $d_{G_{0}}(\Pi(p), \Pi(q)) \leqslant$ $\delta+2 H$. Consider a geodesic $\gamma^{\prime}$ joining $\Pi(p)$ and $\Pi(q)$ in $G_{0}$. Let us assume that $p$ and $q$ belong to the interior of the directed edges $u_{p} v_{p}$ and $u_{q} v_{q}$, respectively (if $p$ or $q$ are vertices the argument is easier). Denote by $w_{p}$ and $w_{q}$ the vertices in $\left\{u_{p}, v_{p}\right\}$ and $\left\{u_{q}, v_{q}\right\}$, respectively, such that $\Pi\left(w_{p}\right), \Pi\left(w_{q}\right) \in \gamma^{\prime}$, and we define $\gamma_{0}^{\prime}$ as the closure of $\gamma^{\prime} \backslash \Pi\left(u_{p} v_{p} \cup u_{q} v_{q}\right)$. Then, there exists a curve $\sigma$ in $G$ from $w_{p}$ to $w_{q}$ such that $\Pi(\sigma)=\gamma_{0}^{\prime}$ and $L_{G}(\sigma) \leqslant L_{G_{0}}\left(\gamma_{0}^{\prime}\right)<d_{G_{0}}(\Pi(p), \Pi(q)) \leqslant \delta+2 H$. We can choose curves $\sigma_{p}, \sigma_{q}$ in $G$ from $p$ to $w_{p}$ and from $w_{q}$ to $q$, respectively, with $L_{G}\left(\sigma_{p}\right), L_{G}\left(\sigma_{q}\right)<2 D(G)$. Then $\sigma_{p} \cup \sigma \cup \sigma_{q}$ is a curve from $p$ to $q$, and $D_{G}(p, q) \leqslant L_{G}\left(\sigma_{p}\right)+L_{G}(\sigma)+L_{G}\left(\sigma_{q}\right) \leqslant \delta+2 H+4 D(G)$. A similar argument gives $D_{G}(q, p) \leqslant \delta+2 H+4 D(G)$.

Therefore, any geodesic triangle $T$ in $G$ is $\delta^{\prime}$-thin, with $\delta^{\prime}=\delta+2 H(\delta, C(G), 4 D(G))+4 D(G)$ and $H$ the constant in Theorem 2.

We prove now one of the main results both of the theory and the applications: the geodesic stability is equivalent to the Gromov hyperbolicity for directed graphs.

Theorem 5. The following statements hold:

I For each $\delta, b, D \geqslant 0$ and $a, C \geqslant 1$, there exists a constant $H=H(\delta, a, b, C, D)$, which only depends on these five parameters, with the following property:

Let us consider any admissible $\delta$-hyperbolic directed graph $G$ with $C(G) \leqslant C$ and $D(G) \leqslant D$, any $x, y \in G$, and any $(a, b)$-quasigeodesic $g$ starting at $x$ and ending at $y$. If $\gamma$ is any geodesic starting at $x$ and ending at $y$ (or starting at $y$ and ending at $x$ ), then $\mathcal{H}_{G}(g, \gamma) \leqslant H$.

II Let us consider any admissible directed graph $G$ with $C(G), D(G)$ finite numbers, satisfying the following property.

For each $a \geqslant 1$ and $b \geqslant 0$ there exists a constant $H$ such that for any $x, y \in G$, any $(a, b)$-quasigeodesic $g$ in $G$ starting at $x$ and ending at $y$, and any geodesic $\gamma$ starting at $x$ and ending at $y$, the inequality $\mathcal{H}_{G}(g, \gamma) \leqslant H$ holds.

Then, $G$ is hyperbolic.

Proof. First of all, note that from (3) we deduce for every $A, B \subseteq G$,

$$
\mathcal{H}_{G}(A, B)-4 D(G) \leqslant \mathcal{H}_{G_{0}}(\Pi(A), \Pi(B)) \leqslant C(G) \mathcal{H}_{G}(A, B) .
$$

Let us prove now the first statement. Consider $x, y \in G$, an $(a, b)$-quasigeodesic $g$ starting at $x$ and ending at $y$, and a geodesic $\gamma$ starting at $x$ and ending at $y$ (or starting at $y$ and ending at $x$ ). According 
to Step 1 in the proof of Theorem 4 we have that $\Pi(\gamma)$ is a $(C, 4 D)$-quasigeodesic in $G_{0}$ joining $\Pi(x)$ and $\Pi(y)$.

We prove now that $\Pi(g)$ is a $(C a, C b+4 D)$-quasigeodesic in $G_{0}$ joining $\Pi(x)$ and $\Pi(y)$. If $g$ : $[\alpha, \beta] \rightarrow G$ is a parametrization of $g$ with $\frac{1}{a}(t-s)-b \leqslant D_{G}(g(s), g(t)) \leqslant a(t-s)+b$ for every $\alpha \leqslant s \leqslant t \leqslant \beta$, then we obtain by using (3)

$$
\frac{1}{a}(t-s)-b-4 D \leqslant d_{G_{0}}(\Pi(g(s)), \Pi(g(t))) \leqslant C a(t-s)+C b \quad \text { for every } \alpha \leqslant s \leqslant t \leqslant \beta .
$$

Therefore, we conclude that $\Pi(g)$ is a $(C a, C b+4 D)$-quasigeodesic in $G_{0}$, since $C \geqslant 1$.

Now, we can finish the proof of the first statement from Definition 9 and Equation (4):

If the directed graph $G$ is $\delta$-hyperbolic, then $G_{0}$ is $\delta$-hyperbolic by Definition 9. According to Theorem 2 there exists a constant $\tilde{H}=\tilde{H}(\delta, C a, C b+4 D) \geqslant 0$ such that if $\gamma_{0}$ is a geodesic in $G_{0}$ joining $\Pi(x)$ and $\Pi(y)$, then $\mathcal{H}_{G_{0}}\left(\Pi(\gamma), \gamma_{0}\right) \leqslant \tilde{H}$ and $\mathcal{H}_{G_{0}}\left(\Pi(g), \gamma_{0}\right) \leqslant \tilde{H} ;$ consequently, $\mathcal{H}_{G_{0}}(\Pi(\gamma), \Pi(g)) \leqslant$ $2 \tilde{H}$. Applying now (4) we deduce $\mathcal{H}_{G}(\gamma, g) \leqslant H$ with $H:=2 \tilde{H}+4 D$.

Let us prove now the second statement. Consider $x_{0}, y_{0} \in G_{0}$, an $(a, 0)$-quasigeodesic $g_{0}$ joining $x_{0}$ with $y_{0}$, and a geodesic $\gamma_{0}$ joining $x_{0}$ with $y_{0}$. Fix $x, y \in G$, with $\Pi(x)=x_{0}$ and $\Pi(y)=y_{0}$. Let us assume that $x$ and $y$ belong to the interior of the directed edges $e_{x}$ and $e_{y}$, respectively, with $\Pi\left(e_{x}\right) \neq$ $\Pi\left(e_{y}\right)$ (if $x$ or $y$ are vertices or $\Pi\left(e_{x}\right)=\Pi\left(e_{y}\right)$, the argument is easier).

If $g_{0}:[\alpha, \beta] \rightarrow G_{0}$ is a parametrization of $g_{0}$ with $\frac{1}{a}(t-s) \leqslant d_{G_{0}}\left(g_{0}(s), g_{0}(t)\right) \leqslant a(t-s)$ for every $\alpha \leqslant s \leqslant t \leqslant \beta$, then we define

$$
\begin{aligned}
& \alpha_{1}:=\sup \left\{t \in[\alpha, \beta] / g_{0}(t) \in \Pi\left(e_{x}\right)\right\}, \\
& \beta_{1}:=\inf \left\{t \in[\alpha, \beta] / g_{0}(t) \in \Pi\left(e_{y}\right)\right\} .
\end{aligned}
$$

As $g_{0}$ is a continuous and injective map, we have $g_{0}(t) \in \Pi\left(e_{x}\right)$ for every $t \in\left[\alpha, \alpha_{1}\right]$, and $g_{0}(t) \in \Pi\left(e_{y}\right)$ for every $t \in\left[\beta_{1}, \beta\right]$.

For each $e \in E\left(G_{0}\right) \backslash\left\{\Pi\left(e_{x}\right), \Pi\left(e_{y}\right)\right\}$ such that $e \cap g_{0}$ has more than one point, we define

$$
\begin{aligned}
& t_{1}^{e}:=\min \left\{t \in[\alpha, \beta] / g_{0}(t) \in e\right\}, \\
& t_{2}^{e}:=\max \left\{t \in[\alpha, \beta] / g_{0}(t) \in e\right\} .
\end{aligned}
$$

As $g_{0}$ is a continuous and injective map, we have $g_{0}\left(t_{1}^{e}\right) \neq g_{0}\left(t_{2}^{e}\right)$ and $g_{0}(t) \in e$ if and only if $t \in\left[t_{1}^{e}, t_{2}^{e}\right]$.

If $t \in\left[\alpha_{1}, \beta_{1}\right]$, then there exists $e \in E\left(G_{0}\right) \backslash\left\{\Pi\left(e_{x}\right), \Pi\left(e_{y}\right)\right\}$ such that $e \cap g_{0}$ has more than one point, with $t \in\left[t_{1}^{e}, t_{2}^{e}\right]$; let us choose a directed edge $g_{0}\left(t_{1}^{e}\right) g_{0}\left(t_{2}^{e}\right) \in E(G)$ and define a curve $g_{2}:\left[\alpha_{1}, \beta_{1}\right] \rightarrow G$ as follows; $g_{2}(t)$ is the unique point in $g_{0}\left(t_{1}^{e}\right) g_{0}\left(t_{2}^{e}\right)$ with $\Pi\left(g_{2}(t)\right)=g_{0}(t)$.

If $e_{x}$ ends at $g_{0}\left(\alpha_{1}\right)$, then we define a curve $g_{1}:\left[\alpha^{\prime}, \alpha_{1}\right] \rightarrow G$ as follows: $g_{1}(t)$ is the unique point in $e_{x}$ with $\Pi\left(g_{1}(t)\right)=g_{0}(t)$, and $\alpha^{\prime}=\alpha$. If $e_{x}$ starts at $g_{0}\left(\alpha_{1}\right)$ and ends, say, at $u$, then let us choose an oriented edge $u g_{0}\left(\alpha_{1}\right) \in E(G)$; we define a curve $g_{1}:\left[\alpha^{\prime}, \alpha_{1}\right] \rightarrow G$ such that $g_{1}$ is an arclength parametrization of $\overline{x u} \cup u g_{0}\left(\alpha_{1}\right)$ (obviously, $\alpha^{\prime}=\alpha_{1}-L_{G}\left(\overline{x u} \cup u g_{0}\left(\alpha_{1}\right)\right)$ ). Note that $\alpha_{1}-\alpha^{\prime}=L_{G}\left(\overline{x u} \cup u g_{0}\left(\alpha_{1}\right)\right)<2 D(G)$.

If $e_{y}$ starts at $g_{0}\left(\beta_{1}\right)$, then we define a curve $g_{3}:\left[\beta_{1}, \beta^{\prime}\right] \rightarrow G$ as follows: $g_{3}(t)$ is the unique point in $e_{y}$ with $\Pi\left(g_{3}(t)\right)=g_{0}(t)$, and $\beta^{\prime}=\beta$. If $e_{y}$ ends at $g_{0}\left(\beta_{1}\right)$ and starts, say, at $v$, then let us choose an oriented edge $g_{0}\left(\beta_{1}\right) v \in E(G)$; we define a curve $g_{3}:\left[\beta_{1}, \beta^{\prime}\right] \rightarrow G$ such that $g_{3}$ is an arclength parametrization of $g_{0}\left(\beta_{1}\right) v \cup \overline{v y}$ (obviously, $\beta^{\prime}=\beta_{1}+L_{G}\left(g_{0}\left(\beta_{1}\right) v \cup \overline{v y}\right)$ ). Note that $\beta^{\prime}-\beta_{1}=L_{G}\left(g_{0}\left(\beta_{1}\right) v \cup \overline{v y}\right)<2 D(G)$.

We prove now that $g:=g_{1} \cup g_{2} \cup g_{3}$ is an $(a C(G), 8 D(G))$-quasigeodesic in $G$ starting at $x$ and ending at $y$. We deal just with the case $\alpha^{\prime}=\alpha_{1}-L_{G}\left(\overline{x u} \cup u g_{0}\left(\alpha_{1}\right)\right)$ and $\beta^{\prime}=\beta_{1}+L_{G}\left(g_{0}\left(\beta_{1}\right) v \cup \overline{v y}\right)$, as the cases $\alpha^{\prime}=\alpha$ and $\beta^{\prime}=\beta, \alpha^{\prime}=\alpha$ and $\beta^{\prime}=\beta_{1}+L_{G}\left(g_{0}\left(\beta_{1}\right) v \cup \overline{v y}\right)$, and $\alpha^{\prime}=\alpha_{1}-L_{G}\left(\overline{x u} \cup u g_{0}\left(\alpha_{1}\right)\right)$ and $\beta^{\prime}=\beta$ are simpler. 
Note that (3) gives

$$
\frac{1}{C(G)} d_{G_{0}}(\Pi(z), \Pi(w)) \leqslant D_{G}(z, w) \leqslant d_{G_{0}}(\Pi(z), \Pi(w))+4 D(G), \quad \text { for every } z, w \in G \text {. }
$$

If $\alpha_{1} \leqslant s \leqslant t \leqslant \beta_{1}$, then (6) gives

$$
\frac{1}{a C(G)}(t-s) \leqslant D_{G}(g(s), g(t)) \leqslant a(t-s)+4 D(G) .
$$

If $\alpha^{\prime} \leqslant s \leqslant t \leqslant \alpha_{1}$ or $\beta_{1} \leqslant s \leqslant t \leqslant \beta^{\prime}$, then

$$
D_{G}(g(s), g(t))=t-s
$$

If $\alpha^{\prime} \leqslant s \leqslant \alpha_{1} \leqslant t \leqslant \beta_{1}$, then as $a, C(G) \geqslant 1$, we deduce

$$
\begin{aligned}
D_{G}(g(s), g(t)) & \leqslant D_{G}\left(g_{1}(s), g\left(\alpha_{1}\right)\right)+D_{G}\left(g\left(\alpha_{1}\right), g_{2}(t)\right) \leqslant 2 D(G)+d_{G_{0}}\left(g_{0}\left(\alpha_{1}\right), g_{0}(t)\right)+4 D(G) \\
& \leqslant a\left(t-\alpha_{1}\right)+6 D(G) \leqslant a(t-s)+6 D(G) \\
D_{G}(g(s), g(t)) & \geqslant D_{G}\left(g\left(\alpha_{1}\right), g_{2}(t)\right)-D_{G}\left(g\left(\alpha_{1}\right), g_{1}(s)\right) \\
& \geqslant \frac{1}{C(G)} d_{G_{0}}\left(g_{0}\left(\alpha_{1}\right), g_{0}(t)\right)-2 D(G) \geqslant \frac{1}{a C(G)}\left(t-\alpha_{1}\right)-2 D(G) \\
& \geqslant \frac{1}{a C(G)}(t-s)-\frac{2 D(G)}{a C(G)}-2 D(G) \geqslant \frac{1}{a C(G)}(t-s)-4 D(G) .
\end{aligned}
$$

If $\alpha_{1} \leqslant s \leqslant \beta_{1} \leqslant t \leqslant \beta^{\prime}$, then a similar argument also gives

$$
\frac{1}{a C(G)}(t-s)-4 D(G) \leqslant D_{G}(g(s), g(t)) \leqslant a(t-s)+6 D(G) .
$$

If $\alpha^{\prime} \leqslant s \leqslant \alpha_{1}$ and $\beta_{1} \leqslant t \leqslant \beta^{\prime}$, then

$$
\begin{aligned}
D_{G}(g(s), g(t)) & \leqslant D_{G}\left(g_{1}(s), g\left(\alpha_{1}\right)\right)+D_{G}\left(g\left(\alpha_{1}\right), g\left(\beta_{1}\right)\right)+D_{G}\left(g\left(\beta_{1}\right), g_{3}(t)\right) \\
& \leqslant 2 D(G)+d_{G_{0}}\left(g_{0}\left(\alpha_{1}\right), g_{0}\left(\beta_{1}\right)\right)+4 D(G)+2 D(G) \\
& \leqslant a\left(\beta_{1}-\alpha_{1}\right)+8 D(G) \leqslant a(t-s)+8 D(G), \\
D_{G}(g(s), g(t)) & \geqslant D_{G}\left(g\left(\alpha_{1}\right), g\left(\beta_{1}\right)\right)-D_{G}\left(g\left(\alpha_{1}\right), g_{1}(s)\right)-D_{G}\left(g_{3}(t), g\left(\beta_{1}\right)\right) \\
& \geqslant \frac{1}{C(G)} d_{G_{0}}\left(g_{0}\left(\alpha_{1}\right), g_{0}\left(\beta_{1}\right)\right)-4 D(G) \geqslant \frac{1}{a C(G)}\left(\beta_{1}-\alpha_{1}\right)-4 D(G) \\
& \geqslant \frac{1}{a C(G)}(t-s)-\frac{4 D(G)}{a C(G)}-4 D(G) \geqslant \frac{1}{a C(G)}(t-s)-8 D(G) .
\end{aligned}
$$

Therefore, we conclude

$$
\frac{1}{a C(G)}(t-s)-8 D(G) \leqslant d_{G_{0}}(\Pi(g(s)), \Pi(g(t))) \leqslant a(t-s)+8 D(G), \quad \text { for every } \alpha^{\prime} \leqslant s \leqslant t \leqslant \beta^{\prime},
$$

and $g$ is an $(a C(G), 8 D(G))$-quasigeodesic in $G$ starting at $x$ and ending at $y$, as $C(G) \geqslant 1$. Furthermore, $g_{0} \subseteq \Pi(g)$ and $L_{G_{0}}\left(\Pi(g) \backslash g_{0}\right) \leqslant D(G)$.

If we replace $g_{0}$ by $\gamma_{0}$, then a similar process also gives an $(a C(G), 8 D(G))$-quasigeodesic $\gamma$ in $G$ starting at $x$ and ending at $y$, with $\gamma_{0} \subseteq \Pi(\gamma)$ and $L_{G_{0}}\left(\Pi(\gamma) \backslash \gamma_{0}\right) \leqslant D(G)$.

Then, by hypothesis, there exists a constant $H$, which depends neither on $g$ or $\gamma(C(G)$ and $D(G)$ are constants which only depends on $G$ ) such that $\mathcal{H}_{G}(\gamma, g) \leqslant H$. By (4), we deduce that $\mathcal{H}_{G_{0}}(\Pi(\gamma), \Pi(g)) \leqslant C(G) \mathcal{H}_{G}(\gamma, g) \leqslant C(G) H$. As $g_{0} \subseteq \Pi(g), \gamma_{0} \subseteq \Pi(\gamma), L_{G_{0}}\left(\Pi(g) \backslash g_{0}\right) \leqslant D(G)$ 
and $L_{G_{0}}\left(\Pi(\gamma) \backslash \gamma_{0}\right) \leqslant D(G)$, we conclude that $\mathcal{H}_{G_{0}}\left(\gamma_{0}, g_{0}\right) \leqslant C(G) H+2 D(G)$. Finally, by Theorem 3, $G_{0}$ is hyperbolic and, by Definition $9, G$ is hyperbolic.

\section{Conclusions}

The theory of hyperbolic metric spaces is a well-studied subject. A natural question is how to develop this theory in the context of spaces with "asymmetric distances". This problem was faced by Edmond Jonckheere in [42] by using noncommutative geometry. Our main aim in this paper is to show a new mathematical theory to provide an alternative and simpler approach to the problem: directed graphs. This point of view introduces asymmetry as something natural. Therefore, in this work, we extend the theory of Gromov hyperbolicity to the new context of directed graphs. In particular, we prove one of the most important results of the theory: a counterpart to the geodesic stability theorem, which happens to be the most utilized theorem both in theory and in applications of hyperbolic spaces in the usual context of geodesic metric spaces.

Author Contributions: The authors contributed equally to this work. All authors have read and agreed to the published version of the manuscript.

Funding: Supported in part by two grants from Ministerio de Economía y Competititvidad, Agencia Estatal de Investigación (AEI) and Fondo Europeo de Desarrollo Regional (FEDER) (MTM2016-78227-C2-1-P and MTM2017-90584-REDT), Spain.

Acknowledgments: We would like to thank the referees for a careful reading of the manuscript and for some helpful suggestions that have improved the presentation of the paper.

Conflicts of Interest: The authors declare no conflicts of interest. The founding sponsors had no role in the design of the study; in the collection, analysis, or interpretation of data; in the writing of the manuscript; or in the decision to publish the results.

\section{References}

1. Gromov, M. Hyperbolic Groups, in Essays in Group Theory; Springer: Berlin, Germany, 1987; pp. 75-263.

2. Ghys, E.; de la Harpe, P. Sur les Groupes Hyperboliques d'après Mikhael Gromov; Birkhäuser: Berlin, Germany, 1990.

3. Bonk, M.; Schramm, O. Embeddings of Gromov hyperbolic spaces. Geom. Funct. Anal. 2000, 10, 266-306. [CrossRef]

4. Foertsch, T.; Schroeder, V. A product construction for hyperbolic metric spaces. Ill. J. Math. 2005, 49, 793-810. [CrossRef]

5. Naor, A.; Peres, Y.; Schramm, O.; Sheffield, S. Markov chains in smooth Banach spaces and Gromov-hyperbolic metric spaces. Duke Math. J. 2006, 134, 165-197. [CrossRef]

6. Wenger, S. Gromov hyperbolic spaces and the sharp isoperimetric constant. Invent. Math. 2008, 171, 227-255. [CrossRef]

7. Aramayona, J. Simplicial embeddings between pants graphs. Geom Dedicata 2010, 144, 115-128. [CrossRef]

8. Holopainen, I.; Soardi, P.M. p-harmonic functions on graphs and manifolds. Manuscripta Math. 1997, 94, 95-110. [CrossRef]

9. Kanai, M. Rough isometries and combinatorial approximations of geometries of non-compact Riemannian manifolds. J. Math. Soc. Jpn. 1985, 37, 391-413. [CrossRef]

10. Portilla, A.; Rodríguez, J.M.; Tourís, E. Stability of Gromov hyperbolicity. J. Adv. Math. Stud. 2009, 2, 1-20.

11. Portilla, A.; Tourís, E. A characterization of Gromov hyperbolicity of surfaces with variable negative curvature. Publ. Mat. 2009, 53, 83-110. [CrossRef]

12. Bowditch, B.H. Notes on Gromov's Hyperobolicity Criterion for Path-Metric Spaces. In Group Theory from a Geometrical Viewpoint; World Scientific: River Edge, NJ, USA, 1991; pp. 64-167.

13. Portilla, A.; Rodríguez, J.M.; Tourís, E. Gromov hyperbolicity through decomposition of metric spaces II. J. Geom. Anal. 2004, 14, 123-149. [CrossRef]

14. Rodríguez, J.M.; Tourís, E. Gromov hyperbolicity of Riemann surfaces. Acta Math. Sin. 2007, 23, 209-228. [CrossRef] 
15. Tourís, E. Graphs and Gromov hyperbolicity of non-constant negatively curved surfaces. J. Math. Anal. Appl. 2011, 380, 865-881. [CrossRef]

16. Bermudo, S.; Carballosa, W.; Rodríguez, J.M.; Sigarreta, J.M. On the hyperbolicity of edge-chordal and path-chordal graphs. Filomat 2016, 30, 2599-2607. [CrossRef]

17. Bermudo, S.; Rodríguez, J.M.; Sigarreta, J.M. Computing the hyperbolicity constant. Comput. Math. Appl. 2011, 62, 4592-4595. [CrossRef]

18. Bermudo, S.; Rodríguez, J.M.; Sigarreta, J.M.; Touris, E. Hyperbolicity and complement of graphs. Appl. Math. Lett. 2011, 24, 1882-1887. [CrossRef]

19. Brinkmann, G.; Koolen, J.; Moulton, V. On the hyperbolicity of chodal graphs. Ann. Comb. 2001, 5, 61-69. [CrossRef]

20. Cantón, A.; Granados, A.; Pestana, P.; Rodríguez, J.M. Gromov hyperbolicity of periodic graphs. Bull. Malays. Math. Sci. Soc. 2016, 39, S89-S116. [CrossRef]

21. Carballosa, W.; de la Cruz, A.; Martínez-Pŕez, A.; Rodríguez, J.M. Hyperbolicity of direct products of graphs. Symmetry 2018, 10, 279. [CrossRef]

22. Frigerio, R.; Sisto, A. Characterizing hyperbolic spaces and real trees. Geom. Dedicata 2009, 142, $139-149$. [CrossRef]

23. Gavoille, G.; Ly, O. Distance Labeling in Hyperbolic Graphs; Springer: Berlin, Germany, 2005; pp. 1071-1079.

24. Granados, A.; Pestana, D.; Portilla, A.; Rodríguez, J.M. Gromov hyperbolicity in Mycielskian Graphs. Symmetry 2017, 9, 131. [CrossRef]

25. Grippo, E.; Jonckheere, E.A. Effective resistance criterion for negative curvature: Application to congestion control. In Proceedings of the 2016 IEEE Conference on Control Applications (CCA), Buenos Aires, Argentina, 19-22 September 2016.

26. Hernández, J.C.; Reyes, R.; Rodríguez, J.M.; Sigarreta, J.M. Mathematical properties on the hyperbolicity of interval graphs. Symmetry 2017, 9, 255. [CrossRef]

27. Jonckheere, E.A.; Lohsoonthorn, P. Geometry of Network Security. In Proceedings of the 2004 American Control Conference, Boston, MA, USA, 30 June-2 July 2004; IEEE: Piscataway, NJ, USA; pp. 111-151. [CrossRef]

28. Jonckheere, E.; Lohsoonthorn, P.; Bonahon, F. Scaled Gromov hyperbolic graphs. J. Graph Theory 2008, 57, 157-180. [CrossRef]

29. Koolen, J.H.; Moulton, V. Hyperbolic Bridged Graphs. Eur. J. Comb. 2002, 23, 683-699. [CrossRef]

30. Méndez-Bermúdez, J.A.; Reyes, R.; Rodríguez, J.M.; Sigarreta, J.M. Hyperbolicity on graph operators. Symmetry 2018, 10, 360. [CrossRef]

31. Rodríguez, J.M.; Sigarreta, J.M.; Vilaire, J.-M.; Villeta, M. On the hyperbolicity constant in graphs. Discret. Math. 2011, 311, 211-219. [CrossRef]

32. Shang, Y. Lack of Gromov-hyperbolicity in colored random networks. Pan Am. Math. J. 2011, 21, 27-36. [CrossRef]

33. Shang, Y. Lack of Gromov-hyperbolicity in small-world networks. Cent. Eur. J. Math. 2012, 10, 1152-1158. [CrossRef]

34. Shang, Y. Random Lifts of Graphs: Network Robustness Based on The Estrada Index. Appl. Math. E Notes 2012, 12, 53-61.

35. Shang, Y. Non-hyperbolicity of random graphs with given expected degrees. Stoch. Model. 2013, 29, 451-462. [CrossRef]

36. Shang, Y. On the likelihood of forests. Phys. A: Stat. Mech. Appl. 2016, 456, 157-166. [CrossRef]

37. Wu, Y.; Zhang, C. Hyperbolicity and chordality of a graph. Electr. J. Combin. 2011, 18, 43.

38. Charney, R. Artin groups of finite type are biautomatic. Math. Ann. 1992, 292, 671-683. [CrossRef]

39. Baryshnikov, Y. On the Curvature of the Internet; Workshop on Stochastic Geometry and Teletraffic: Eindhoven, The Netherlands, 2002.

40. Jonckheere, E.; Lohsoonthorn, P.; Ariaei, F. Upper bound on scaled Gromov hyperbolic delta. Appl. Math. Comput. 2007, 192, 191-204. [CrossRef]

41. Kleinberg, R. Geographic routing using hyperbolic space. In Proceedings of the IEEE INFOCOM 2007-26th IEEE International Conference on Computer Communications, Barcelona, Spain, 6-12 May 2007; IEEE: Piscataway, NJ, USA, 2007; pp. 1902-1909. [CrossRef] 
42. Jonckheere, E.A. Contrôle du trafic sur les réseaux à géometrie hyperbolique-Une approche mathématique a la sécurité de l'acheminement de l'information. J. Eur. Syst. Autom. 2003, 37, 145-159.

43. Hespanha, J.P.; Bohacek, S. Preliminary Results in Routing Games. In Proceedings of the 2001 American Control Conference, Arlington, VA, USA, 25-27 June 2001. [CrossRef]

44. Jonckheere, E.A.; Lohsoonthorn, P. A hyperbolic geometry approach to multi-path routing. In Proceedings of the 10th Mediterranean Conference on Control and Automation (MED 2002), Lisbon, Portugal, 9-12 July 2002; FA5-1.

45. Anderson, J.W. Hyperbolic Geometry; Springer: London, UK, 1999.

46. Chen, B.; Yau, S.-T.; Yeh, Y.-N. Graph homotopy and Graham homotopy. Discret. Math. 2001, 241, $153-170$. [CrossRef]

47. Bonk, M. Quasi-geodesics segments and Gromov hyperbolic spaces. Geom. Dedicata 1996, 62, $281-298$. [CrossRef]

48. Hausdorff, F. Set Theory; American Mathematical Society (English translation): Providence, RI, USA, 1957.

49. Künzi, H.P.A. Nonsymmetric Distances and Their Associated Topologies: About the Origins of Basic Ideas in the Area of Asymmetric Topology. In Handbook of the History of General Topology; Kluwer Academic Publishers: Boston, MA, USA, 2001; Volume 3, pp. 853-968.

50. Schellekens, M. The Smyth completion: A common foundation for denotational semantics and complexity analysis. Electr. Notes Theor. Comp. Sci. 1995, 1, 211-232. [CrossRef]

51. García-Raffi, L.M.; Romaguera, S.; Sánchez-Pérez, E.A. Sequence spaces and asymmetric norms in the theory of computational complexity. Math. Comp. Modell. 2002, 36, 1-11. [CrossRef]

(C) 2020 by the authors. Licensee MDPI, Basel, Switzerland. This article is an open access article distributed under the terms and conditions of the Creative Commons Attribution (CC BY) license (http:/ / creativecommons.org/licenses/by/4.0/). 